\title{
Fabrication and Characterization of CTS/Coconut Composite Membranes Crosslinked with Glutaraldehyde for Elimination of Heavy Metal in Aqueous Solution
}

\author{
Truong Thi Cam Trang1* ${ }^{*}$ Nguyen Quang Huy², Takaomi Kobayashi ${ }^{3}$ \\ ${ }^{1}$ Faculty of Environment Science, VNU-HCM University of Science, Ho Chi Minh City, Vietnam \\ ${ }^{2}$ Department of International Cooperation, Ministry of Natural Resources and Environment, Hanoi, Vietnam \\ ${ }^{3}$ Department of Science of Technology Innovation, Nagaoka University of Technology, \\ Niigata, Japan \\ Email: *ttctrang@hcmus.edu.vn
}

How to cite this paper: Trang, T.T.C., Huy, N.Q. and Kobayashi, T. (2019) Fabrication and Characterization of CTS/Coconut Composite Membranes Crosslinked with Glutaraldehyde for Elimination of Heavy Metal in Aqueous Solution. Journal of Agricultural Chemistry and Environment, 8, 69-77.

https://doi.org/10.4236/jacen.2019.82006

Received: August 25, 2018

Accepted: April 1, 2019

Published: April 4, 2019

Copyright $\odot 2019$ by author(s) and Scientific Research Publishing Inc. This work is licensed under the Creative Commons Attribution International License (CC BY 4.0).

http://creativecommons.org/licenses/by/4.0/

\begin{abstract}
Chitosan/coconut (CTS/coconut) composite membranes were successfully prepared by the cross-linking reaction with glutaraldehyde and they were applied in eliminating heavy metals from aqueous solutions. The cross-linked membranes were obtained at the ratios of $1 / 1,1 / 1.5$ and $1 / 2$ and the coconut fiber was chemically treated by $\mathrm{NaOCl} / \mathrm{NaOH}$. The best ratio of CTS/coconut fiber is found to be $1 / 1.5$ which has a relatively high stability with the degree of swelling (DS) and solvent content (SC) of membrane to be $13.33 \%$ and $69.88 \%$, respectively. The results also indicate that the CTS membranes showed preferential separation of heavy metals for blend CTS/coconut membranes.
\end{abstract}

\section{Keywords}

Crab Shell, Membrane Technology, Chitosan, Coconut Fiber, Heavy Metal

\section{Introduction}

Today, trace metal pollution from industry is a serious threat to human health and the ecosystems due to toxicity, too vague and bioaccumulation in natural [1]. Therefore, the application of treatment methods to reduce or eliminate heavy metals in water is crucial [2].

So far, a number of efficient methods have been reviewed for the removal of heavy metals such as chemical precipitation, ion exchange, reverse osmosis, electrodialysis, ultrafiltration, nanofiltration, coagulation, flocculation, floatation, 
etc. [3]. However, these methods have several disadvantages such as high reagent, unpredictable metal ion removal, generation of toxic sludge, etc. Adsorption process very simple, effective and versatile has become one of the most appropriate techniques, but this method has disadvantages such as small capacity, high cost, difficulty for large-scale application, etc. In 2011, a report showed the removal of some heavy metals $\mathrm{Cu}(\mathrm{II}), \mathrm{Cd}(\mathrm{II}), \mathrm{Mn}(\mathrm{II}), \mathrm{Pb}(\mathrm{II}), \mathrm{As}(\mathrm{III})$ and $\mathrm{As}(\mathrm{V})$ from aqueous solution using adsorption and nano-filtration membrane techniques [4] [5] [6]. The adsorbents may be made of mineral, organic or biological origin: activated carbons, zeolites, clay, silica beads, low-cost adsorbents (industrial by-products, agricultural wastes, biomass) and polymeric materials (organic polymeric resins, macroporous hyper crosslinked polymers) are significant examples [7] [8] [9]. In addition, as biopolymers are being used, Chitosan (CTS) is a material that can be found in crustacean shells, which is usually wasted by seafood industries. Therefore, as a suitable biopolymer, their applications for blended and composite membranes have been reported. It is known that CTS is a cyclo-aliphatic polymer that contains both active amino groups and hydroxyl groups. By using CTS cross-linked membranes by several chemicals such as glutaraldehyde, formaldehyde, sulfuric acid, therefore, the CTS/coconut membranes PV performance for elimination of heavy metals for water was investigated [10] [11] [12].

\section{Experiments}

\subsection{Chemicals and Reagents}

Crab shells were purchased from Vung Tau City, Vietnam and coconut fiber was collected in Wood production Company, Binh Dinh Province, Vietnam. Hydrochloric acid, Sodium hydrocid, Sodium hydroxide, Ethanol and Acetic acid were purchased from China. Solutions of Cd (II), Cr (VI) ions were prepared by dissolving weighed quantities of $3 \mathrm{CdSO}_{4} \cdot 8 \mathrm{H}_{2} \mathrm{O}$ (China), $\mathrm{K}_{2} \mathrm{Cr}_{2} \mathrm{O}_{7}$ (Merck, Germany). All chemicals were of analytical reagent grade. Deionizer water was used for dilutions and reagent preparations. Salt solutions containing heavy metals were prepared in the laboratory at specific concentrations.

\subsection{Membrane Preparation}

\subsubsection{Preparation of Chitosan from Crab Shells}

Crab shells were washed with water and dried in 8 hours at $105^{\circ} \mathrm{C}$ and then cut into small scrabs. Extracting Chitosan from crab shells was in 5 steps namely: demineralization, deproteinization, decolorization $1^{\text {st }}$, deacetylation and decolorization $2^{\text {nd }}$. Crab shells were placed in flask and $\mathrm{HCl}$ solution was added in the ratio of (1:10) stirred for 12 hours for demineralization. After that, the deproteinization stage was conducted by mixing crab shells with $\mathrm{NaOH}$ at $1 / 10$ ratio (wt/v) for 12 hours. Crab shells were decolored with Ethanol at $50^{\circ} \mathrm{C}$ in 5 hours. The deacetylation stage was conducted by stirring crab shells with $\mathrm{NaOH} 40 \%$ wt with $1 / 10$ ratio $(\mathrm{wt} / \mathrm{v})$ at $80^{\circ} \mathrm{C}$ in 6 hours. Finally, crab shells were stirred with 
acid acetic $10 \mathrm{wt} \%$ for 24 hours and centrifuged to obtain Chitosan, Chitosan was added $\mathrm{NaOH} 40 \mathrm{wt} \%$ to $\mathrm{pH}=10$ to be decolored again.

\subsubsection{Preparation of Chitosan/Coconut Membrane Composite}

The coconut fiber was washed with water then cut into $(0.5-1 \mathrm{~cm})$. Coconut was stirred with $\mathrm{NaOCl}$ and $\mathrm{NaOH}$ for decolorization. The investigation was conducted in 4 steps as illustrated in Figure 1.

Figure 1 shows the CTS/coconut membranes composite preparation, the membranes were obtained as follows: Chitosan was dissolved in Acetic acid 2 wt $\%$. Then, $100 \mathrm{~mL}$ Chitosan solution $2 \mathrm{wt} \%$ was mixed with coconut in other ratios and stirred for 24 hours. $10 \mathrm{~mL}$ Glutaraldehyde and $10 \mathrm{~mL} \mathrm{HCl} 0.5 \mathrm{M}$ were added to this solution as a crosslinking. After the process was finished, the homogeneous solution was poured into a flat glass plate and CTS/coconut membranes were formed after dried at room temperature for 24 hours. Three different membranes for the CTS/coconut mixtures were prepared as ratio of CTS/coconut fiber at $1 / 1 ; 1 / 1.5$ and $1 / 2(\mathrm{v} / \mathrm{wt})$ respectively.

\subsection{CTS/Coconut Membranes Composite Characterization}

Scanning electron microscopy (SEM) was used to study the morphology of the cross-section of the CTS/coconut membranes composite (By JSM-5300L V (JEOL. Japan)).

Fourier transform infrared spectroscopy (FT-IR) was recorded by grinding dried CTS/coconut fiber samples with potassium bromide ( $\mathrm{KBr})$ in the transmittance model (By JASCO FT-IR/4100 spectrometer). The spectra were taken within $4000-400 \mathrm{~cm}^{-1}$ wavenumber.

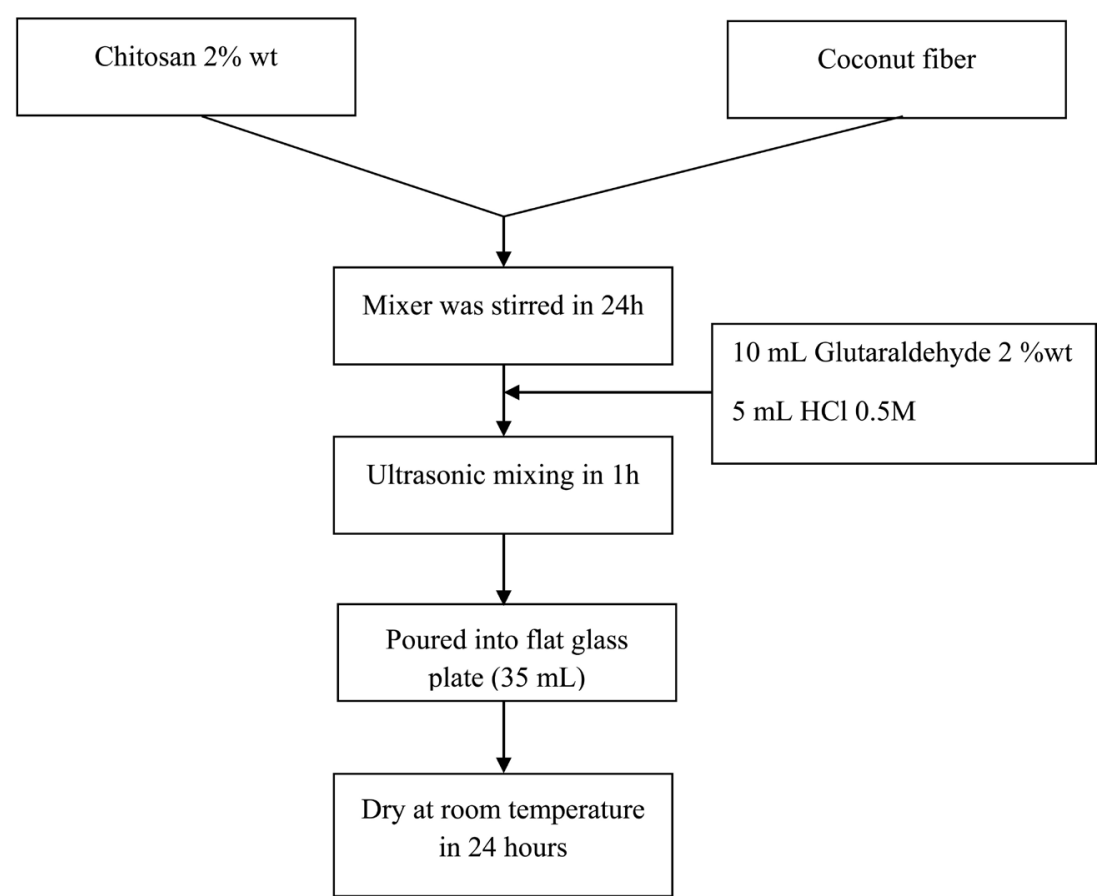

Figure 1. Schematic procedure of preparation of CTS/coconut membranes composite. 
Solvent content was determined from the difference in the weight of the membranes before and after immersion in water. The solvent content $(S C)$ was expressed as:

$$
S C=\left[\left(W_{s}-W_{d}\right) / W_{d}\right] \times 100 \%
$$

where $W_{d}$ and $W_{s}$ denote the weight of the films before and after immersion in solution, respectively.

The degree of swelling $(D S)$ of the CTS/coconut membranes was calculated by comparing the volume of the membranes before $(V b)$ and after $(V a)$ immersion in the solution:

$$
D S=V(a) / V(b)
$$

\subsection{The Experiment of Heavy Metal Removal}

The experiment was conducted to investigate the stability of heavy metal removal by CTS/coconut membranes composite. The membrane with the optimal ratio CTS/coconut fiber was chosen for this experiment.

As shown in Figure 2, heavy metal solution was prepared with determined concentration of Cd (II) and Cr (VI). The filtration process was conducted by adding $40 \mathrm{~mL}$ heavy metal sample into the model. After that, turn on the pump to let the flow continue through CTS/coconut membranes composite in 1 hour (the pump was used with the lowest pressure). Output solution was placed in glass bottle and analyzed.

\section{Results and Discussion}

\subsection{CTS/Coconut Membranes}

Figure 3 shows the CTS/coconut membranes composite with different ratios CTS/coconut fiber at (b) $1 / 1$, (c) $1 / 1.5$ and (d) $1 / 2$. The membranes were sclerosed, brown or yellow. The various color of membrane depends on the ratios of CTS/coconut fiber. The membrane had brown color when the ratio of coconut increase and had yellow color when the ratio of Chitosan increases.

\subsection{Characteristics of CTS/Coconut Membranes}

As mentioned in the experimental section, membranes composite consisting of

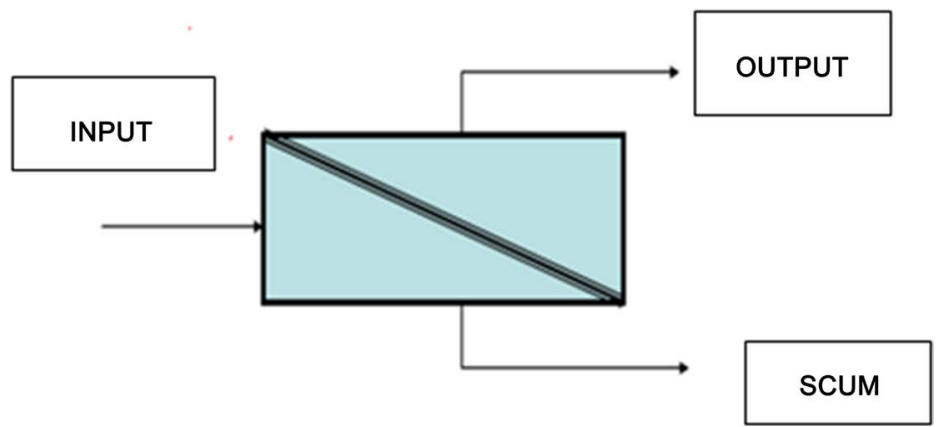

Figure 2. Schematic of heavy metals removal by CTS/coconut model. 
CTS and coconut fiber were prepared with different ratio of CTS and coconut fiber at 1/1,1/1.5 and 1/2. Each membrane was cross-linked by omiting Glutaraldehyde and Hydrochloric acid. In order to choose the optimal ratio CTS/coconut fiber, the solvent content (SC) and the degree of swelling (DS) were examined. Figure 4 shows the resultant SEM images of the CTS/coconut membranes. In

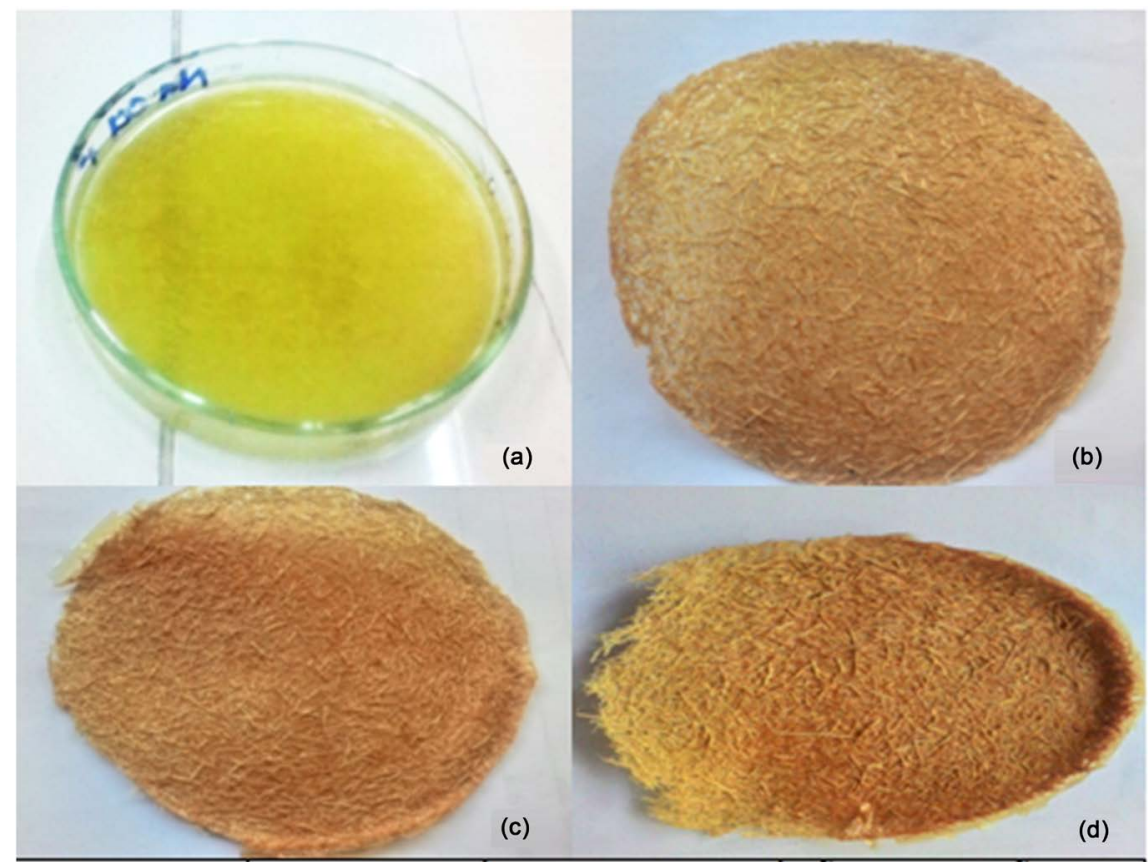

Figure 3. The images of CTS/coconut membranes composite with different ratios at 1/1, 1/1.5 and 1/2.

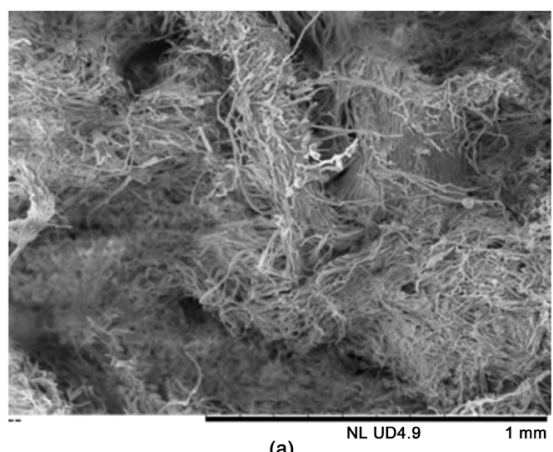

(a)

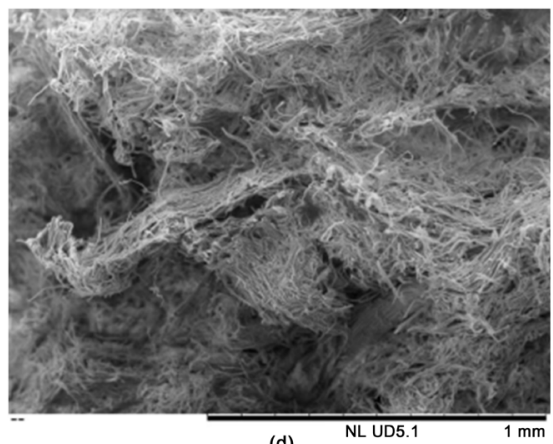

(d)

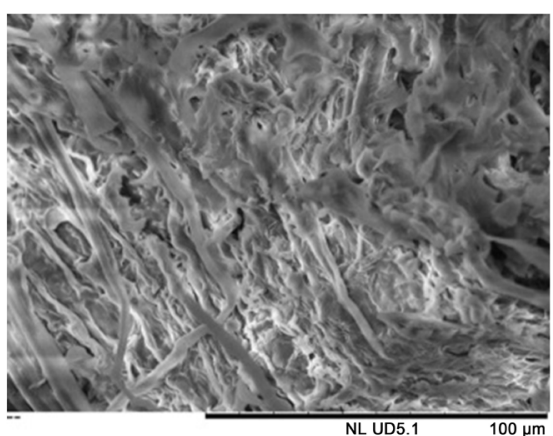

(b)

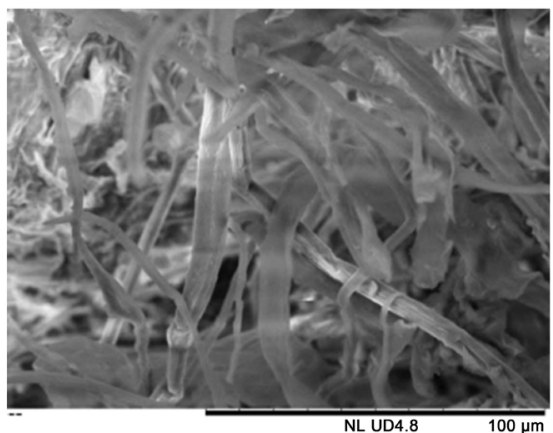

(e)

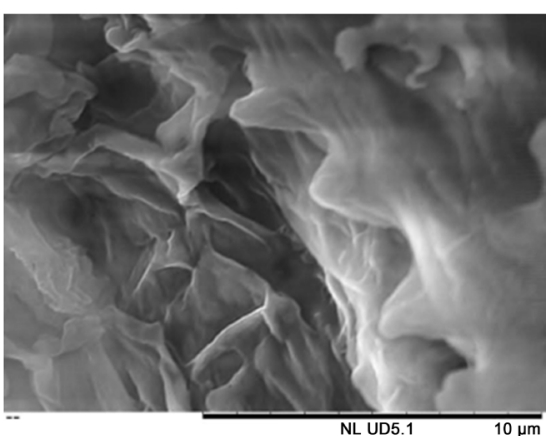

(c)

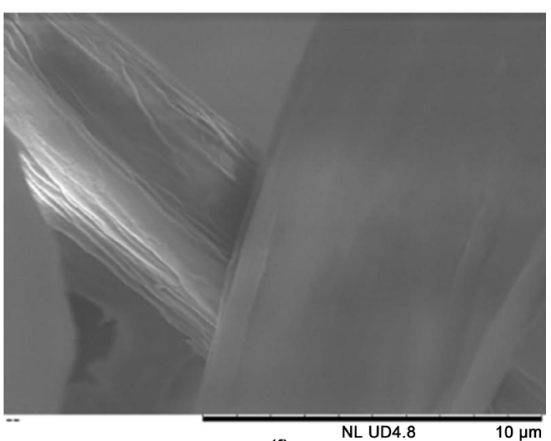

Figure 4. SEM images of CTS/coconut membranes (a-c) Surface of CTS/coconut membrane (a) $\times 100$ (b) $\times 1000$ (c) $\times 10,000$, $((d)-(f))$ Cross-section of CTS/coconut membranes (d) $\times 100$ (e) $\times 1000(f) \times 10,000$. 
the pictures $((\mathrm{d})-(\mathrm{f}))$, the cross section of the obtained membranes that the material is covered. The membrane presented many coconut fiber having about 10 $\mu \mathrm{m}$ in presented many CTS/coconut fiber that the morphological structure of the surfaces of the four membranes in the picture ((a)-(c)) was obtained with the cross-linking treatment.

Figure 5 shows the DS of CTS/coconut membranes composite increased in first hour and became stable after 2 hours. The DS of the membranes with ratio CTS/coconut fiber at $1 / 2(11.67 \%)$ was lower than the ratios at $1 / 1(15 \%)$ and $1 / 1.5(13.33 \%)$. That was due to the stable linking of coconuts fiber in membrane make the CTS could not swell in water.

The solvent content (SC) reflects the ability to absorb water, which affects the stability of the membranes and the ability to process heavy metal ion in water.

Based on the graph (Figure 6), the solvent content (SC) of CTS/coconut membranes composite increased over time but predominantly in the first hour, then continued slowly in 24 hours. This might be explained by the solvent content of chitosan and coconut fiber in the membranes. The ratio CTS/coconut fiber at $1 / 1.5(70 \%)$ was lower than the ratios CTS/coconut fiber at $1 / 1(82 \%)$ and $1 / 2(75 \%)$.

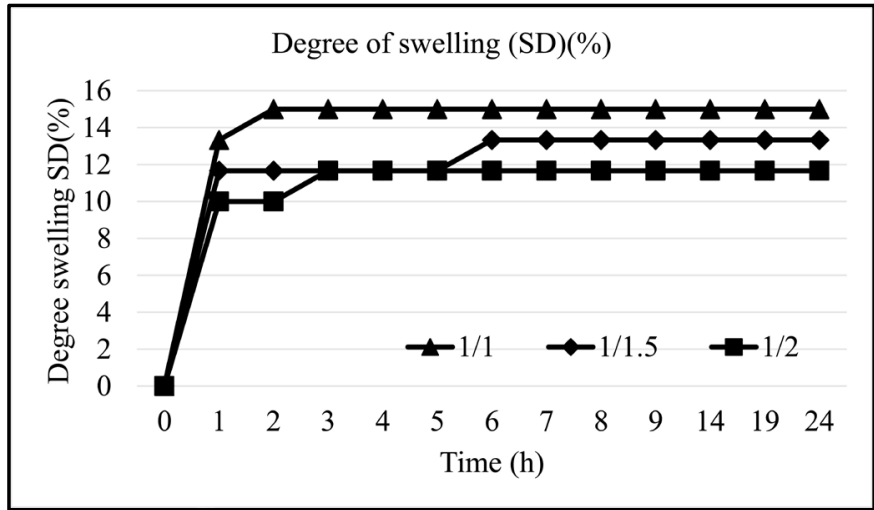

Figure 5. The degree of swelling (DS) of CTS/coconut membranes composite at ratios $1 / 1,1 / 1.5$ and $1 / 2$ after 24 hours.

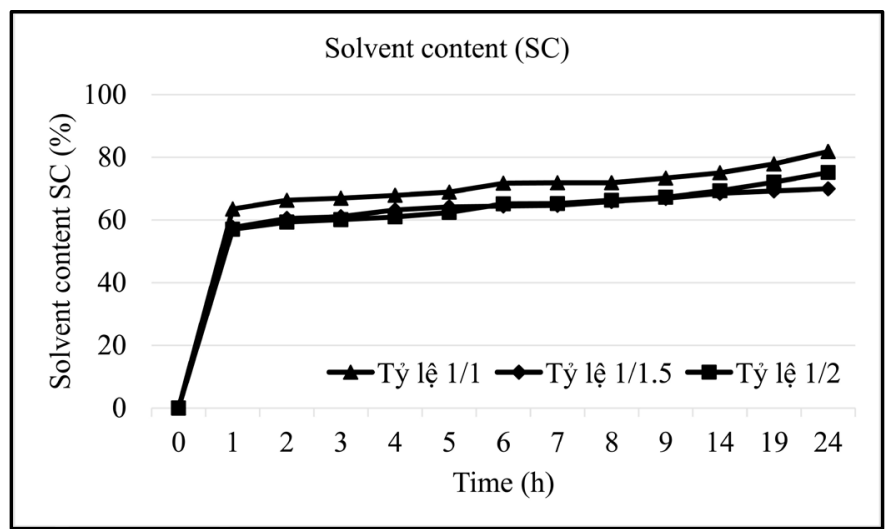

Figure 6. The solvent content (SC) of SC of CTS/coconut membranes composite at ratios $1 / 1,1 / 1.5$ and $1 / 2$ after 24 hours. 
Based on the solvent content (SC) and the degree of swelling (DS), the optimal ratio $\mathrm{CTS} /$ coconut fiber is $1 / 1.5$ ratio.

\subsection{The Experiment of Heavy Metal Removal}

Figure 7 and Figure 8 show the results of the efficiency of the chromium removal and cadimium in single solutions and in the ternary system. As shown from these results, the chromium removal was higher than the cadimium removal in both systems.

This result could be explained that the CTS/coconut membrane composite exists $-\mathrm{NH}_{2}$ and $-\mathrm{OH}$, which are capable of forming complex substances with heavy metals. The preference of heavy metals in complexing might be based on the HSAB theory (Pearson's classification). According to HSAB, the hard acid will link to hard bases. Besides, Chromium is a known hard acid and $-\mathrm{OH}$ as a hard base, in solution, $-\mathrm{OH}$ and Chromium created a strong bond and was preferred over $-\mathrm{NH}_{2}$ with soft acid.

In addition, in a CTS/CF membrane, $-\mathrm{NH}_{2}$ and $-\mathrm{OH}$ groups exist. These radicals are capable of complexing with heavy metals. The mechanism for complexing with metals is shown in Figure 9. In addition, the relatively high metal content of the water combined with the hydrophilic nature of the membrane results in competition between metal ions and water molecules as they pass through the membrane, causing space constraints. When this occurs, metal ions don't bond

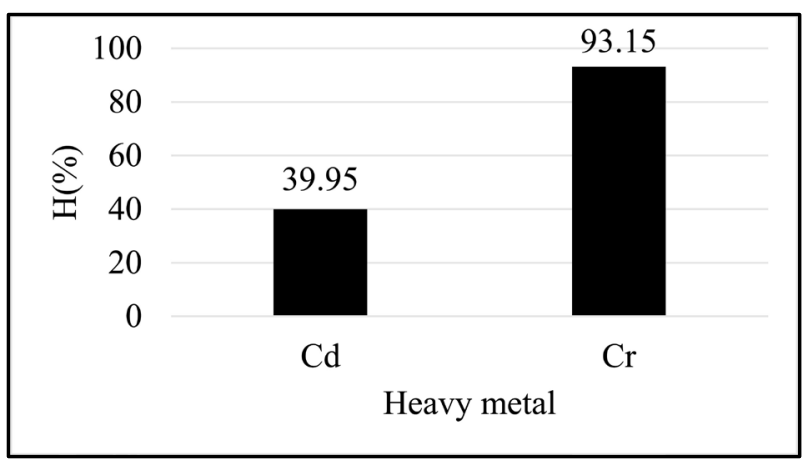

Figure 7. The efficiency of heavy metal removal by CTS/coconut membranes composite in single solutions.

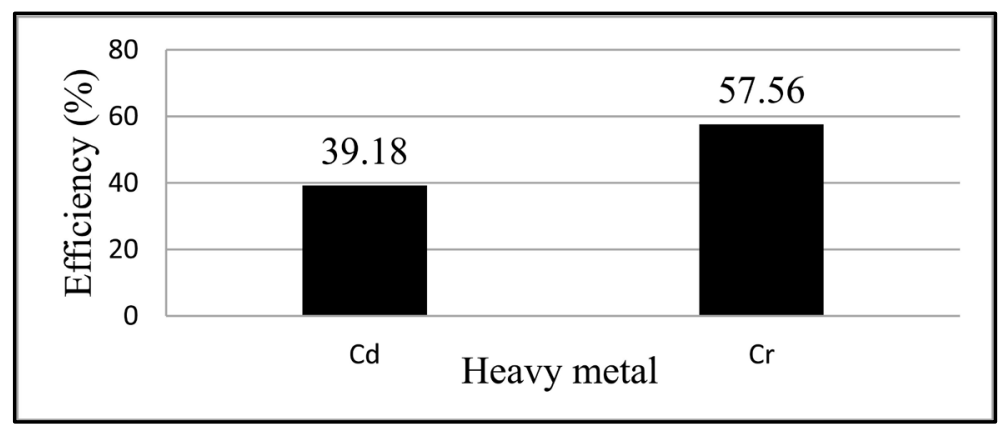

Figure 8. The efficiency of heavy metal removal by CTS/coconut membranes composite in the ternary system. 


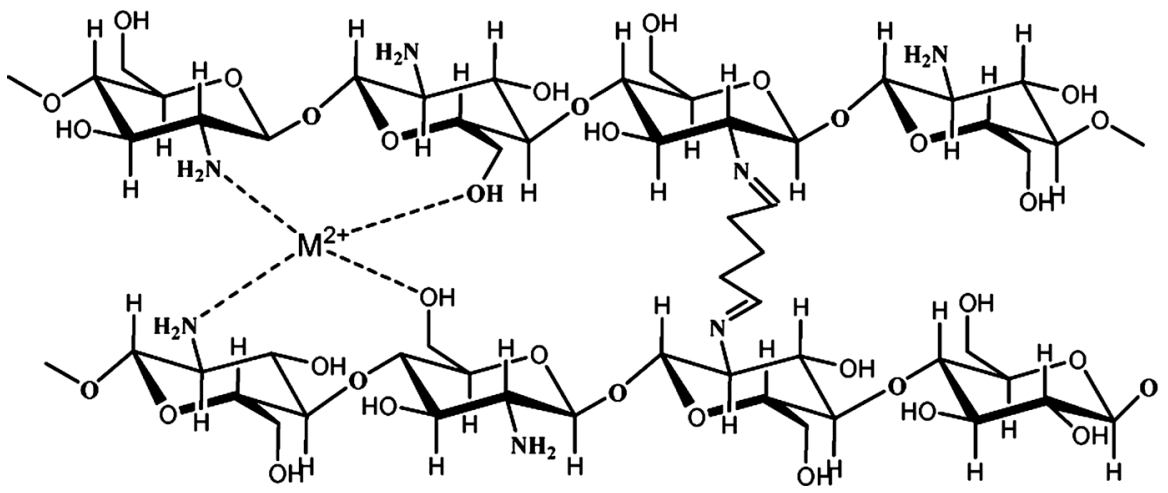

Figure 9. The structure of the complex mechanism of CTS/coconut membranes composite with cations.

with $-\mathrm{NH}_{2}$ and $-\mathrm{OH}$ groups, resulting in poor separation efficiency. The interaction of metal ions such as $\mathrm{Cr}$, $\mathrm{Cd}$ with the $-\mathrm{NH}_{2}$ groups in polymer molecules can occur, and their resulting hydrated radiuses are large. This makes it more difficult for them to pass through the membrane than other ions. The diffusion of metal ions through the membrane is also affected by the distribution of the cellulose constituents in the coconut fiber network, the structural tightness, the size selection and the shape of structure, resulting in variable separation efficiencies seen in different metal.

In addition, the heavy metal removal efficiency might be explained by the hydrophilicity of the membranes. In solution, water molecules competed with heavy metal ions and reduced the potential for contact of the heavy metal ions with $-\mathrm{OH}$ and $-\mathrm{NH}_{2}$.

\section{Conclusion}

We have succeeded in synthesizing CTS/coconut membranes composite capable of treating some heavy metals in water of the optima; CTS/ coconut fiber ratio is $1 / 15$. In addition, investigation of CTS/coconut membranes composite properties and the heavy metals removal showed that CTS/coconut membranes composite was the best treatment for $\mathrm{Cr}$ (VI) metal contaminated water.

\section{Acknowledgements}

This research is funded by Vietnam National University Ho Chi Minh City (VNU-HCM) under grant number C2016-18-05.

\section{Conflicts of Interest}

The authors declare no conflicts of interest regarding the publication of this paper.

\section{References}

[1] Huang, Y.-C. and Sefa Koseoglu, S. (1993) Separation of Heavy Metals from Industrial Waste Streams by Membrane Separation Technology. Waste Management, 13, 481-501. https://doi.org/10.1016/0956-053X(93)90079-C 
[2] Baker, R.W. (2000) Membrane Technology and Applications. McGraw-Hill, New York City, 1-84.

[3] Ayisha Begum, A., Radhakrishnan, R. and Prem Nazeer, K. (2011) Study of Structure-Property Relationship on Sulfuric Acid Crosslinking Chitosan Membranes. Malaysian Polymer Journal, 6, 27-38.

[4] Brígida, A.I.S., Calado, V.M.A., Gonçalves, L.R.B. and Coelho, M.A.Z. (2010) Effect of Chemical Treatments on Properties of Green Coconut Fiber. Carbohydrate Polymers, 79, 832-838. https://doi.org/10.1016/j.carbpol.2009.10.005

[5] Nigiz, F.U. and Hilmioglu, N.D. (2013) Evaporation of Ethanol/Water Mixtures by Zeolite Filled Sodium Alginate Membrane. Desalination and Water Treatment, 51, 637-643. https://doi.org/10.1080/19443994.2012.714582

[6] Moon, G.Y. (2000) Synthesis and Preparation of Polysaccharide Based Membranes for the Evaporation of Liquid Mixture Systems of Industrial Interest. National Library of Canada, 76-170.

[7] Abdul Khalil, H.P.S., Siti Alwani, M. and Mohd Omar, A.K. (2006) Chemical Composition, Anatomy, Lignin Distribution, and Cell Wall Structure of Malaysian Plant Waste Fibers. BioResources, 1, 220-232.

[8] Sudhakar, H., Maruthi, Y., Sajan Kumarji Rao, U., et al. (2013) Improved Evaporation Performance of 13X Zeolite Filled Chitosan Membranes. Indian Journal of Advances in Chemical Science, 2, 21-31.

[9] An, H.K., No, H.K. and Kim, D.S. (2001) Comparison of Heavy Metal Ions $\left(\mathrm{Pb}^{2+}\right.$, $\left.\mathrm{Cd}^{2+}, \mathrm{Mn}^{2+}, \mathrm{Cu}^{2+}\right)$ Removal by Several Materials. Environmental Engineering Research, 6, 117-122.

[10] Ostrowska-Czubenko, J. (2009) Effect of Ionic Cross Linking on the Water State in Hydrogel Chitosan Membranes. Carbohydrate Polymers, 77, 590-598.

https://doi.org/10.1016/j.carbpol.2009.01.036

[11] Pierog, M., Gierszewska, M. and Ostrowska, J. (2009) Effect of Ionic Cross-Linking Agents on Swelling Behavior of Chitosan Hydrogel Membranes. Progress on Chemistry and Application of Chitin, 14, 75-82.

[12] Shukla, P.M. and Shukla, S.R. (2013) Biosorption of $\mathrm{Cu}(\mathrm{II}), \mathrm{Pb}(\mathrm{II}), \mathrm{Ni}(\mathrm{II})$, and Fe(II) on Alkali Treated Coir Fibers. Separation Science and Technology, 48, 421-428. https://doi.org/10.1080/01496395.2012.691933 